\title{
Reacción granulomatosa a cuerpo extraño en punta y dorso nasal por materiales de relleno. Presentación de un caso clínico y revisión de la literatura
}

\section{Granulomatous foreign body reaction in tip and nasal dorsum due to filling materials. Presentation of a clinical case and review of the literature}

Pablo Pérez $\mathbf{R}^{1}$, Alejandra Villarroel $\mathbf{P}^{2}$, Tomás Andrade $\mathbf{D}^{3}$, Gabriel Faba $\mathbf{C}^{3}$.

\begin{abstract}
RESUMEN
Paciente de 29 años con antecedente de tres rinomodelaciones en la punta nasal con ácido hialurónico hace cinco años, evaluada por deseo de mejora estética nasal y obstrucción nasal. Al examen visual se observó una leve giba osteocartilaginosa, punta bulbosa, narinas simétricas, sin laterorrinea, no se palparon masas ni nodulaciones. Posterior al manejo de su rinitis y previo consentimiento informado, al realizar rinoseptoplastía abierta se encontró gran cantidad de tejido granulatorio tipo cuerpo extraño en la punta y dorso nasal lo que obligó a cambiar el plan quirúrgico. La biopsia diferida confirmó granuloma por cuerpo extraño por biopolímero derivado de la silicona. Evolucionó satisfactoriamente estética y funcionalmente. La rinomodelación ha aumentado en los últimos años, aunque no existen rellenos faciales aprobados por la FDA para uso nasal. La correcta utilización de los rellenos nasales continúa siendo un tema controvertido. Los granulomas nasales por cuerpo extraño son complicaciones tardías que se han incrementado con el uso de biopolímeros con dosis y técnicas inadecuadas. El uso de ecotomografía ha contribuido a mejorar la planificación quirúrgica, así como el estudio histológico diferido ha permitido identificar el material utilizado en la mayoría de los casos convirtiéndose en el estándar de oro.
\end{abstract}

Palabras clave: Rellenos nasales, rinomodelación.

\begin{abstract}
A 29-year-old patient with a history of three nasal fillings in the nasal tip with hyaIuronic acid five years ago, reason for consultation improvement nasal aesthetics and nasal obstruction. Physical examination showed a slight osteocartilaginous hump, bulbous tip, symmetrical nostrils, no deviation nasal pyramid, no masses or nodules were palpated. After the management of his rhinitis and previous informed consent, when performing open rhinoseptoplasty, a large amount of foreign body type granulation tis-

\footnotetext{
Departamento Otorrinolaringología, Pontifica Universidad Católica de Chile, Santiago, Chile.

2 Servicio Anatomía Patológica, Pontificia Universidad Católica UC Christus, Santiago, Chile.

3 Comité de Nariz, Departamento Otorrinolaringología, Pontifica Universidad Católica de Chile, UC Christus, Santiago, Chile.

* Los autores declaran no tener conflictos de interés.
}

Recibido el 18 de abril, 2018. Aceptado el 3 de julio, 2018. 
sue was found in the tip and nasal dorsum, which forced the surgical plan to change. The post-surgical biopsy showed foreign body granuloma by biopolymer derived from silicone. Evolved satisfactorily aesthetically and functionally. The use of nasal fillers has increased in recent years, although there are no facial fillers approved by the FDA for nasal use. The correct use of nasal fillers is a controversial issue. Nasal granulomas by foreign body are late complications that have increased with the use of biopolymers with inadequate doses and techniques. The use of ecotomography has contributed to improve the surgical planning; the deferred histological study has allowed to identify the material used in most cases becoming the gold standard

Key words: Nasal fillers, rhinomodelation, granuloma.

\section{INTRODUCCIÓN}

Los materiales de relleno son productos de origen orgánico o sintéticos que tienen como finalidad la corrección de defectos de volumen con fines tanto reconstructivos como estéticos ${ }^{1}$. En las últimas décadas, ha sido en este último ámbito donde se han empleado con más frecuencia, especialmente en la zona facial $\left.\right|^{2}$, sin embargo, aún no existe un relleno ideal que cumpla con todas las características de ser una sustancia segura para el organismo, biocompatible, no antigénico, no pirogénico, estable tras su administración, de extracción fácil (si fuera preciso), no tóxico, no carcinogénico y de costo razonable ${ }^{3}$. La FDA tiene autorizados más de 20 tipos de materiales de rellenos para la región perinasal, su uso en rinomodelación no está autorizado por esta entidad. Su utilización está basada en la experiencia clínica que se ve en reportes 0 serie de $\operatorname{cas}^{4}{ }^{4,5}$. Estos materiales, si bien la mayoría de las veces producen poca morbilidad, no están exentos de riesgos al ser administrados ${ }^{6,7}$. En el caso de los granulomas por cuerpo extraño secundarios a rinomodelación, éstos pueden aparecer por cualquier material de relleno utilizado, en un plazo variable de 5 meses a 15 años, siendo más frecuente que ocurran al ser utilizados con técnicas y dosis inadecuadas y con el uso de materiales sintéticos de mayor duración ${ }^{8,9}$. Su prevención, identificación precoz y manejo continúa siendo un tema de controversia siendo además difícil su manejo quirúrgico.

\section{CASO CLÍNICO}

Se presenta el caso de una paciente de sexo femenino de 29 años, sin antecedentes mórbidos ni cirugías previas. No fumadora. Consultó en el servicio de otorrinolaringología por obstrucción nasal y corrección estética nasal. Tenía el antecedente de tres infiltraciones con ácido hialurónico en la punta nasal hace 5 años. Al examen físico se observó un dorso ancho en su tercio medio, con leve giba osteocartilaginosa, punta bulbosa, sin laterorrinia y narinas simétricas. A la palpación no se identificaron masas ni induraciones (Figura 1).

Se realizó manejo médico de su rinitis con antialérgicos orales y corticoides nasales con buena respuesta. Debido a que la paciente consultaba además por cirugía nasal estética para definir y angostar su punta nasal, se planificó realizar una rinoseptoplastía abierta previo consentimiento informado. Durante la cirugía al exponer la punta nasal se encontró una gran cantidad de tejido tipo cuerpo extraño con reacción granulomatosa en punta y dorso nasal de consistencia firme, adherido a piel y tejido osteocartilaginoso, poco vascularizado lo que obligó a cambiar el plan quirúrgico inicial (Figura 2).

El dorso nasal se encontraba comprometido en su porción osteocartilaginosa sin infiltrar la piel, se pudo encontrar un plano de clivaje para su extracción completa. En la punta nasal el tejido infiltraba los cartílagos alares en su porción lateral y la piel. No se pudo encontrar un plano de clivaje que permitiera diferenciar el límite entre el tejido normal y el granuloma por lo que se decidió dejar escasa cantidad de tejido en punta nasal para no comprometer la vascularización de esta zona y prevenir necrosis cutánea. El resultado de la biopsia diferida informó la presencia de tejido conectivo con inflamación granulomatosa, compatible con una reacción de tipo cuerpo extraño por biopolímero derivado de la silicona (Figura 3 ).

No se observó un patrón histológico compatible con ácido hialurónico, el que habría sido el material 


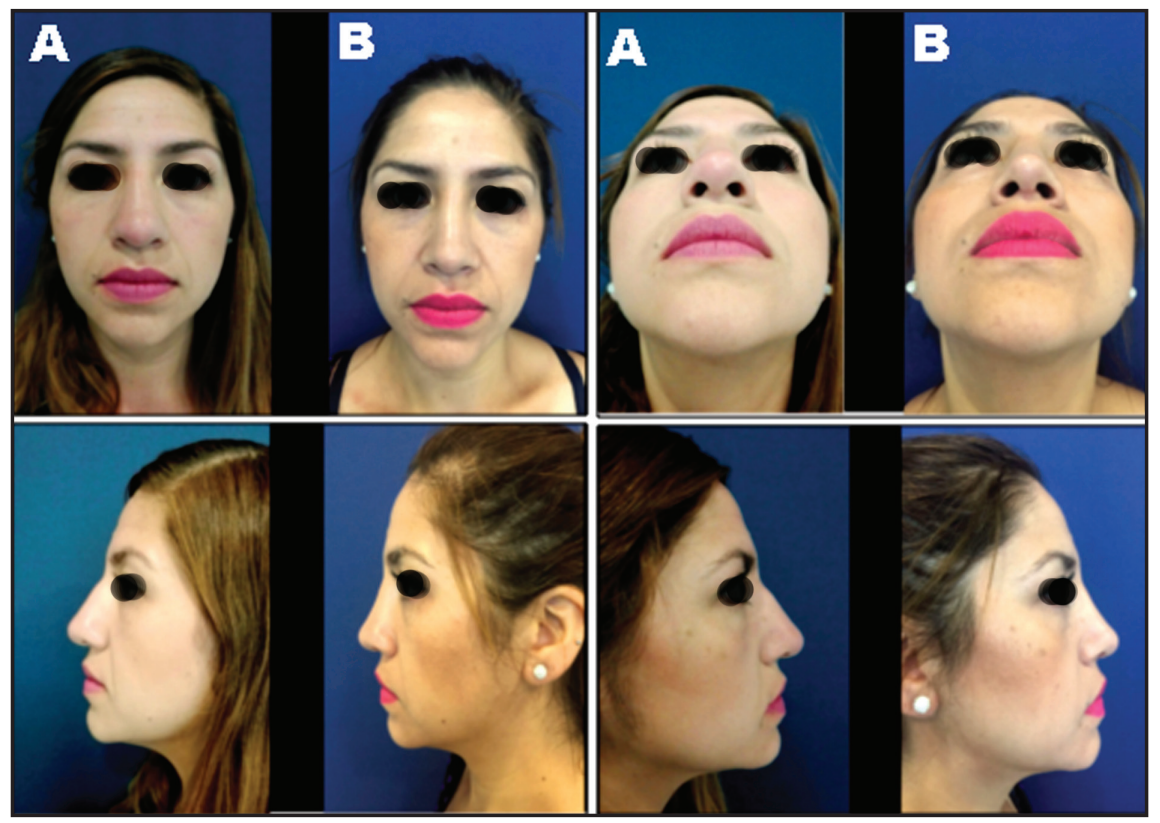

Figura 1. Se observan visiones comparativas preoperatorias (A) a la izquierda y posoperaraorias (B) a la derecha a los 30 días.

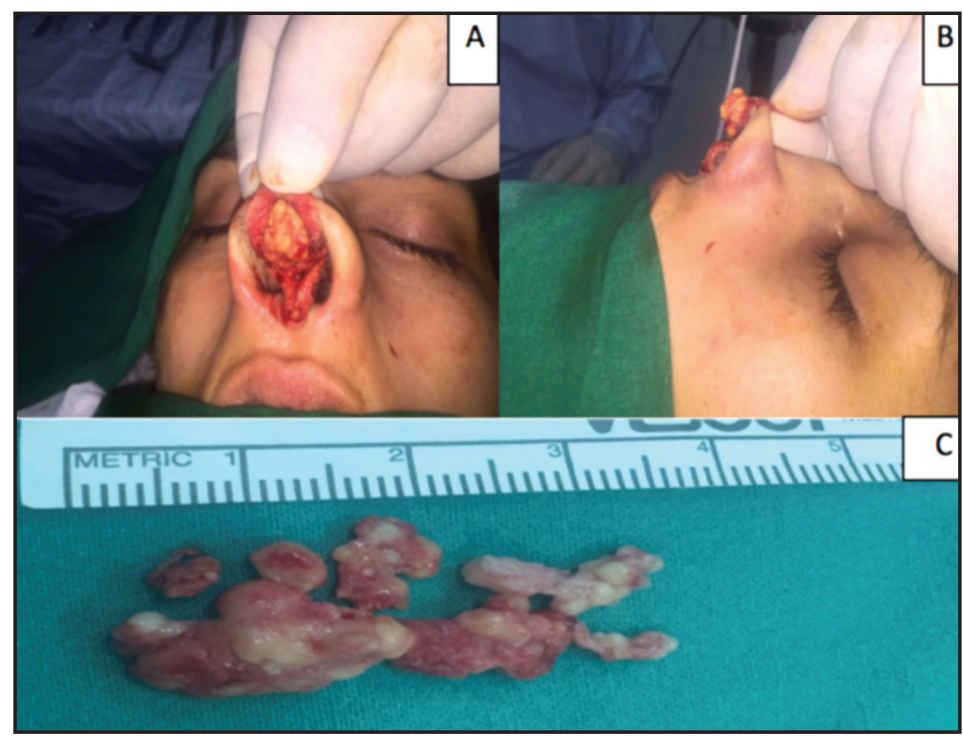

Figura 2. Reacción granulomatosa tipo cuerpo extraño. (A) Se observa el compromiso en cartílagos laterales inferiores (alares) y dorso nasal. (B) Se observa la infiltración en piel, sin un plano de disección en punta nasal. (C) Pieza quirúrgica con tejido granulomatoso resecado.

de relleno usado según los antecedentes referidos por la paciente. Durante el posoperatorio inmediato evolucionó de manera satisfactoria, sin complica- ciones. En control de los 30 días posoperada se observó una punta nasal definida, narinas simétricas, dorso recto y tabique nasal alineado (Figura 1). 
Figura 3. Cortes histológicos de pieza quirúrgica (tinción hematoxilina-eosina). (A) Se observa tejido conectivo (1) y cartílago hialino (2) de arquitectura conservada adyacente a tejido conectivo con inflamación de aspecto granulomatoso (3). (B) Se identifican células gigantes de tipo reacción a cuerpo extraño (flecha) y espacios redondeados ópticamente vacíos de tamaño variable (asterisco) (HE, 4x). (C) Se observa con mayor resolución células gigantes de tipo reacción a cuerpo extraño (flechas) y espacios ópticamente vacíos y heterogéneos "en queso suizo", característicos de reacción por cuerpo extraño por biopolímeros de los derivados de la silicona (HE, 10x).

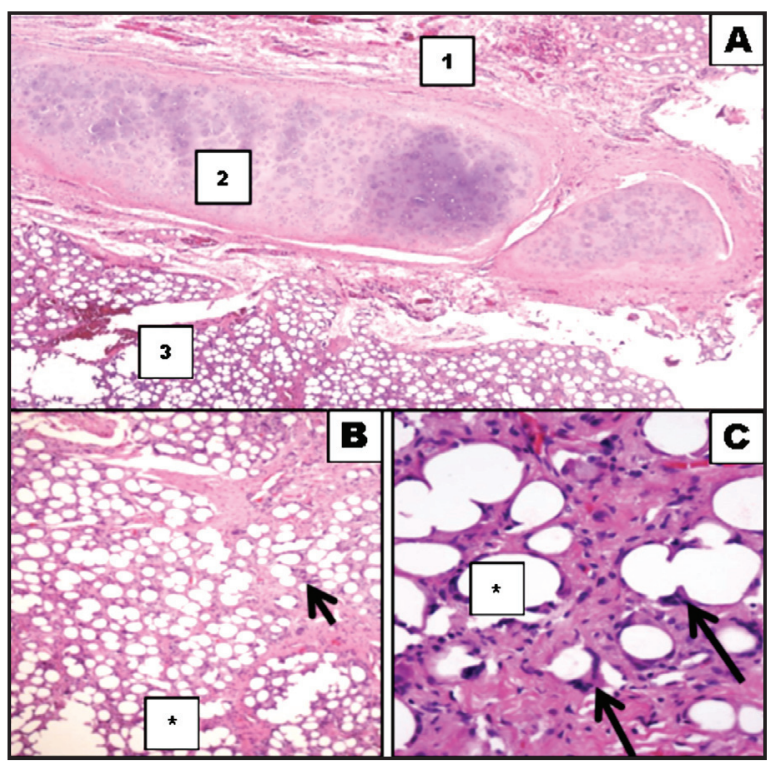

áreas faciales. Se recomienda que sea profunda, directamente sobre el pericondrio o periostio con el fin de evitar complicaciones vasculares como embolias y necrosis de piel dentro de otras ${ }^{10-12,16}$.

El material de relleno más utilizado es el ácido hiaIurónico, debido a su baja antigenicidad y fácil mezcla en los tejidos, sin embargo, puede requerir mayores volúmenes para lograr el efecto deseado y presenta una reabsorción entre 4 a 6 meses según el tipo ${ }^{3,17}$. Existe un creciente uso de materiales sintéticos 0 biopolímeros (ácido poli-L-láctico, hidroxiapatita de calcio, polimetilmetacrilato, gel de poliacrilamida, silicona) que permiten mayor resistencia a deformación y requieren un menor volumen de inyección con una duración mayor dependiendo del material utilizado

Tabla 1. Estudios publicados en rinomodelación en general, se debe considerar su utilización en línea media tanto en dorso como en punta nasal para evitar la infiltración vascular del material de relleno, inyección lenta y considerar la aplicación de presión lateral a los sitios de inyección para evitar la migración del material ${ }^{5,10,12,14}$. La viscosidad del material afectará la profundidad con la que se debe inyectar el material. Los materiales más viscosos deben inyectarse de forma más superficial mientras que los menos viscosos pueden ser utilizados en mayor profundidad ${ }^{15}$. Se debe destacar que la técnica de infiltración en la punta nasal es distinta a las otras

\begin{tabular}{|lll|}
\hline Autor & Año & \multicolumn{1}{c|}{ Diseño } \\
\hline Thomas & 2016 & Revisión \\
Moon & 2016 & Revisión \\
Heden & 2016 & Reporte de casos \\
Jallut & 2014 & Revisión y reporte de caso \\
Stupak & 2007 & Cohorte prospectiva \\
Dayan & 2007 & Revisión \\
Gurney & 2007 & Revisión \\
Beer & 2006 & Serie de casos \\
Cohen & 2005 & Revisión y reporte de caso \\
Hobar & 2000 & Revisión \\
Karwowsky & 1955 & Reporte de caso \\
\hline
\end{tabular}


llegando incluso a los 6 años. No obstante, se han reportado mayor cantidad de reacciones adversas a cuerpo extraño tipo granuloma, en comparación al ácido hialurónico ${ }^{3}$. La silicona se encuentra prohibida por la FDA desde 1991 para su uso inyectable en cualquier parte del cuerpo por su asociación con reacciones autoinmunes, infección, hematoma, formación de granulomas por cuerpo extraño, migración a tejidos vecinos y difusión tanto hacia el plano dérmico como al músculo-cutáne03-5,7,18,19. Independiente del material de relleno utilizado todos pueden presentar efectos adversos siendo los más frecuentes durante las primeras semanas luego de su administración. Ejemplos a considerar son los cambios de pigmentación, prurito, dolor, edema, equimosis, exceso de corrección, etc ${ }^{3,6,7,17}$. Reacciones a largo plazo se presentan desde los 5 meses hasta años después de su uso. Incluyen la migración del material, la degradación a sustancias tóxicas y/o inmunogénicas, reacciones inflamatorias y/o granulomatosas, formación de queloides, despigmentación, embolización, extrusión, disestesias entre otras $3,6,7,10,17$. Todos los materiales de relleno inducen reacciones de cuerpo extraño pudiendo evolucionar hacia granulomas entre los 5 meses y 15 años después de su uso ${ }^{3,20}$. Se presentan clínicamente como induraciones no dolorosas en el lugar de inyección, también pueden presentarse en sitios distantes por migración. Se presentan en menor frecuencia cuando se usan materiales que se reabsorben en menor tiempo como el ácido hialurónico.

El uso de biopsia diferida se considera el estándar de oro para diferenciar la naturaleza de los distintos materiales de relleno utilizados ${ }^{20-22}$. Además nos permite descartar otros procesos inflamatorios, infecciosos (tuberculosis, leishmaniasis) y neoplási$\cos ^{23}$. El estudio histológico ha cambiado el requisito tradicional de biocompatibilidad, ha demostrado que todos los materiales de relleno producen algún tipo de reacción tisular que puede ser subclínica, es decir no son inertes y presentan distintos grados de bioactividad ${ }^{24}$. En el caso de nuestra paciente la

\section{BIBLIOGRAFÍA}

1. Fernández S. Respuesta tisular a materiales de relleno estudio experimental. Tesis Doctoral Universidad de Compostela 2007; 7: 37 (297).

2. American Society of Plastic Surgeons. 2016 presencia de células gigantes multinucleadas con espacios vacíos heterogéneos Figuras $3 b$ y $3 c$ nos permitió descartar la presencia de ácido hialuróni$\mathrm{CO}^{21,22}$. El metacryl y la silicona presentan espacios vacíos, pero a diferencia de los derivados de la silicona éstos son homogéneos y con luz polarizada se observan las partículas en suspensión ${ }^{22}$. El uso de ecotomografía de partes blandas en pacientes con antecedente de rinomodelación es una herramienta de apoyo en el seguimiento clínico y en caso de complicaciones es una ayuda en la planificación quirúrgica, ya que permite determinar los planos comprometidos de manera más exacta que el examen clínico, evaluar si existe compromiso vascular y determinar el grado de migración del material. Si bien no permite realizar una tipificación exacta del material, permite descartar por exclusión de patrón el tipo de material utilizado ${ }^{25-27}$. Tampoco permite realizar una medición del material, en el caso de existir calcificaciones disminuye su capacidad de evaluar los planos comprometidos 26,27 .

\section{CONCLUSIÓN}

Se debe tener en mente varios planes quirúrgicos y realizar el adecuado a cada caso adaptándose en la misma cirugía. Tomando en cuenta las múltiples variables a considerar la técnica abierta es la más utilizada dado que permite una mayor exposición del granuloma. Considerar eso sí que en algunos casos pudiera haber compromiso severo de la piel, con eventual alteración de la circulación, por lo que en algunos casos sería de elección la técnica cerrada para evitar necrosis de la piel. Creemos que la ecotomografía de partes blandas debe ser usado en caso de antecedente de rinomodelación debido a que permite anticipar potenciales riesgos de la cirugía, así como informar mejor al paciente de su condición prequirúrgica, sus riesgos y abordar las expectativas de mejor manera.

Plastic Surgery Statistics Report. https:// www. plasticsurgery.org/documents/News/ Statistics/2016/plastic-surgery-statistics-fullreport-2016.pdf. Visitado en abril 2018.

3. Donald W, Buck II, Murad A. Injectable fillers for facial rejuvenation: A review. Journal of Plastic, 
Reconstructive and Aesthetic Surgery 2009; 62 : 11-8.

4. United States Food and Drug Administration. Dermal Fillers Approved by the Center for Devices and Radio- logical Health http://www.fda.gov/ MedicalDevices/ProductsandMedicalProcedures/ Cosmetic Devices/Wrinkle Fillers/ucm227749. htm. Visitado en Marzo 6, 2017.

5. Michael E, Jasin M. Nonsurgical Rhinoplasty Using Dermal Fillers. Facial Plast Surg Clin N Am 2013; 21: 241-52.

6. Wollina U, Goldman A. Dermal fillers: Facts and controversies. Clinics in Dermatology 2013; 31: 731-6.

7. Woodward J, Khan T, Martin J. Facial Filler Complications. Facial Plast Surg Clin N Am 2015; 23: 447-58.

8. Min LeE J, KIm Y. Foreign Body Granulomas after the Use of Dermal Fillers: Pathophysiology, clinical Appearance, histologic features, and Treatment. Arch Plastic Surgery 2015; 42: 232-9.

9. Grunebaum LD, Bogdan Allemann I, Dayan S et al. The risk of alar necrosis associated with dermal filler injection. Dermatol Surg 2009; 35: 1635-40.

10. Jallut Y, et al. Nguyen Rhinoplasty and dermal fillers. Annales de chirurgie plastique esthétique 2014; 542-7.

11. Michael E et al. Nonsurgical Rhinoplasty Using Dermal Fillers. Facial Plast Surg Clin N Am 2013; 21: 241-52.

12. Hedén P at al. Nasal Reshaping with Hyaluronic Acid: An Alternative or Complement to Surgery. Plast Reconstr Surg Glob Open 2016; 4: e1120.

13. Siclovan R, Jamal A. Injectable Calcium Hydroxyapatite for Correction of Nasal Bridge Deformities. Aesth Plast Surg 2009; 33: 544-8.

14. Tamer $F$ et al. Non-surgical minimally invasive rhinoplasty: tips and tricks from the perspective of a dermatologist. Act Dermatovenerol 2017; 26: 101-3.

15. Pierre S, Liew S, Bernardin A. Basics of Dermal Filler Rheology. Dermatologic Surgery2015; 41: 12-126.
16. Kurkjian T, Ahmad J, Rohrich R. Soft-Tissue Fillers in Rhinoplasty. Plastic and Reconstructive Surgery 2014; 133: 121e-126e.

17. Leo L, Friedman 0. Update on injectables in the nose. Curr Opin Otolaryngol Head Neck Surg 2017; 25: 307-13.

18. Paul E et al. The History of Injectable Silicone Fluids for Soft-Tissue Augmentation. American Society of Plastic Surgeons. Plast Reconstr Surg 2007; 120: 2034-40.

19. Mary D, Anderson L, Alun R. Wang-Silicone migration and granuloma formation. Journal of Cosmetic Dermatology 2009; 8: 92-7.

20. Requena L et at. Adverse reactions to injectable soft tissue fillers. J Am Acad Dermatol 2011; 64: 1-34.

21. Ferneini E, Beauvais D, Steven I. An Overview of Infections Associated with Soft Tissue Facial Fillers: Identification, Prevention, and Treatment. Journal of Oral and MaxilloFacial Surgery 2017; 75: 160-6.

22. Mohamed El-Khalawany et al. Dermal filler complications: a clinic pathologic study with a spectrum of histologic reaction patterns. Annals of Diagnostic Pathology 2015; 19: 10-5.

23. IZQUIERDO M, REQUENA L. Foreign body granulomas. Actas Dermo sifilográficos 1999; 90: 543-57.

24. Funt D, Pavicic T. Dermal fillers in aesthetics: an overview of adverse events and treatment approaches. Clin Cosmet Investing Dermatol 2013; 6: 295-316.

25. Mlosek RK, Skrzypeke, Skrzypek DM, Malinowska S. High-frequency ultrasound-based differentiation between nodular dermal filler deposits and foreign body granulomas. Skin Res Technol 2018; 24: 417-22.

26. De Cabo-Francés F, Alcolea J, Bové-Farré I, Pedret C., Trelles M.A. Ultrasound of injecable fillers and its role in diagnosis tracking. Cir Plast Iberolatinoam 2012; 38: 179-87.

27. Schelke L, Elzen H, Erkamp P, Neumann H. Use of ultrasound to provide overall information on facial fillers and surrounding tissue. Dermatol Surg 2010; 36: 1843-51.

\footnotetext{
Correspondencia: Pablo Esteban Pérez Rivas Marcoleta 350, Santiago, Chile

E mail : dr.pabloperez.rivas@gmail.com
} 